\section{Ultra-high-sensitivity two-dimensional bend sensor}

G.A. Cranch, G.M.H. Flockhart, W.N. MacPherson, J.S. Barton and C.K. Kirkendall

\begin{abstract}
A multicore fibre Fabry-Perot-based strain sensor interrogated with tandem interferometry for bend measurement is described. Curvature in two dimensions is obtained by measuring the difference in strain between three co-located low finesse Fabry-Perot interferometers formed in each core of the fibre by pairs of Bragg gratings. This sensor provides a responsivity enhancement of up to 30 times that of a previously reported fibre Bragg grating based sensor. Strain resolutions of $0.6 \mathrm{n} \varepsilon / \mathrm{Hz}^{1 / 2}$ above $1 \mathrm{~Hz}$ are demonstrated, which corresponds to a curvature resolution of $\sim 0.012 \mathrm{~km}^{-1} / \mathrm{Hz}^{1 / 2}$.
\end{abstract}

Introduction: Multiple point measurement of curvature is a requirement for the realisation of smart materials with embedded shape sensors. The fibre-optic Bragg grating sensor is well suited for this application and numerous demonstrations of embedded strain sensor arrays have been reported [1]. Multicore fibre (MCF)-based Bragg grating curvature sensors have been demonstrated to provide highperformance two-dimensional curvature measurement [2]. Highresolution differential strain measurements of $6 \mathrm{n} \varepsilon / \mathrm{Hz}^{1 / 2}$ can be achieved using interferometric interrogation [3] and has recently been applied to MCF FBG curvature sensors [4]. This type of fibre Bragg grating (FBG) curvature sensor has the added advantage of being compatible with wavelength and time division multiplexing [5]. We report a further enhancement of this sensing concept, which provides at least an order of magnitude enhancement in the resolution of the curvature sensor. The proposed sensor is based on low finesse, Bragg-grating-based, Fabry-Perot interferometers formed in the MCF and uses tandem interferometry to address each sensor.

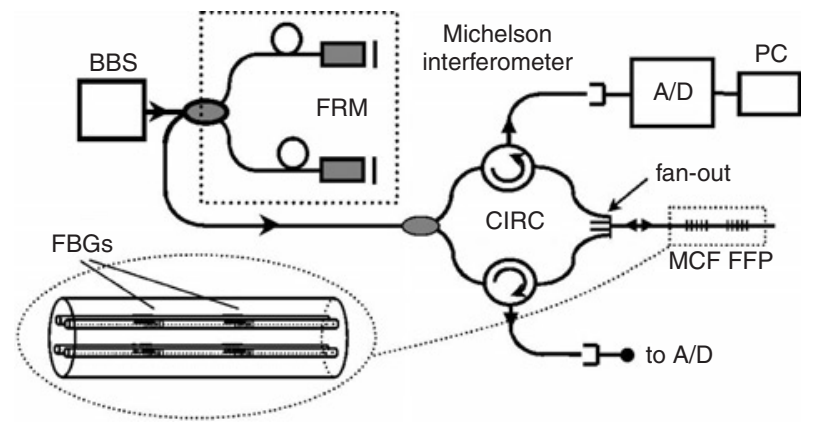

Fig. 1 Experimental setup

For illustrative purposes, interrogation of only two fibre cores in MCF shown

Sensor arrangement: The sensor arrangement is shown in Fig. 1. The output of a broadband erbium fibre amplifier source (BBS) is launched into a chirped FBG (not shown), which narrows the spectrum to about $2 \mathrm{~nm}$. The reflection from the chirped FBG is amplified and launched into a fibre Michelson interferometer (MI) with a fibre path imbalance of $6.1 \mathrm{~cm}$. Faraday rotation mirrors (FRM) are used in the MI to remove effects of birefringence in the MI fibre. The output of the MI is split into three (only two are shown in Fig. 1, the third is used to interrogate the third fibre core in the same manner) using directional couplers and each is passed through a circulator and launched into a core of the MCF, through a fan-out of the type described in [2]. A pair of FBGs separated by $6.1 \mathrm{~cm}$, have been formed in each core of the MCF, as shown in the inset of Fig. 1. The FBGs were written with a frequency-doubled argon laser at $244 \mathrm{~nm}$ with the MCF positioned in the near field of a phase mask. They were written individually, by rotating the fibre to align with each core prior to exposure. The reflectivities of the FBGs making up the fibre Fabry-Perot interferometers (FFP) are sufficiently low such that the response resembles that of a two beam interferometer. The MCF is a cloverleaf design of the type used in [6]. The FBGs are $\sim 3 \mathrm{~mm}$ in length, exhibit reflectivities over the range $0.8-5.5 \%$, and Bragg wavelengths between 1550.4 and $1551.0 \mathrm{~nm}$. In our experimental system, the fan-out exhibits a back reflection large enough to significantly degrade the optical signal-to-noise ratio of the FFP signal. On detection, this broadband back reflection beats with the signal reflected from the FBGs, generating excess noise. Thus narrowing the bandwidth of the input radiation reduces the backreflected light and hence this noise source. Improvement in the fabrication of the fan-out can eliminate this back-reflection, removing the need to narrow the spectrum at the input.

The total phase of each Tandem interferometer corresponding to core $m$ is given by,

$$
\phi_{m}=4 \pi / \lambda_{m} \cdot\left(n_{M I} L_{M I}-n_{m} L_{m}\right)
$$

where $\lambda_{m}$ is the Bragg wavelength of the FBGs, $n_{M I}$ is the refractive index of the fibre making up the MI, $L_{M I}$ is the fibre path imbalance of the MI, and $n_{m}$ and $L_{m}$ are the effective refractive index and the fibre length, respectively, of the Fabry-Perot cavity in core $m$. The phase shift corresponding to FFP cavity $m$ is then given by,

$$
\begin{aligned}
\Delta \phi_{m}= & \left(4 \pi / \lambda_{m}\right)\left[\zeta n_{M I} \Delta L_{M I}+L_{M I} \xi_{M I} \Delta T_{M I}\right] \\
& -\left(4 \pi / \lambda_{m}\right)\left[\zeta n_{m} \Delta L_{m}+L_{m} \xi_{m} \Delta T_{m}\right] \\
& -\left(2 \pi O P D_{m} / \lambda_{m}^{2}\right) \Delta \lambda_{m}
\end{aligned}
$$

where $\zeta$ is the stress-optic coefficient and is equal to $\sim 0.78$, $\xi=\partial n /\left.\partial T\right|_{\rho}$ is the thermo-optic coefficient, which is assumed to be the same for each core, $O P D_{m}=2 n_{M I} L_{M I}-2 n_{m} L_{m}$ is the effective optical path imbalance, $\Delta T_{M I}$ and $\Delta T_{m}$ are the temperature change of the MI and MCF-FFP cavity, respectively, and $\Delta L$ is a length change. Assuming that $L_{m}=L_{m+1}$ and $O P D_{m}=0$, then the difference in phase between the two cavities is

$$
\Delta \phi_{m}-\Delta \phi_{m+1}=4 \pi \zeta\left[\left(n_{m} / \lambda_{m}\right) \Delta L_{m}-\left(n_{m+1} / \lambda_{m+1}\right) \Delta L_{m+1}\right]
$$

In practice, the refractive index of the cores may not be equal, in which case the differential length change between two cores can be calculated from $4 \pi \zeta\left[\Delta L_{m}-\Delta L_{m+1}\right] \simeq\left(\lambda_{m} / n_{m}\right) \Delta \phi_{m}-\left(\lambda_{m+1} / n_{m+1}\right) \Delta \phi_{m+1}$. Thus, the difference phase is related only to the changes in length $\Delta L_{m}$ and $\Delta L_{m+1}$ of the two FFPs and phase changes due to environmental effects on the MI are nominally cancelled. If the fibre is subjected to uniform curvature, then the differential strain is related to the curvature by $\Delta \varepsilon=\left(\Delta L_{m}-\Delta L_{m+1}\right) / L_{m}=d / R$ where $d$ is the core spacing $(=50 \mu \mathrm{m}$ for this MCF) and $R$ is the radius of curvature. The curvature is defined as $1 / R$. Taking $\lambda_{B}=1550.5 \mathrm{~nm}, n=1.468$, and $L=6.1 \mathrm{~cm}$ yields a responsivity, $\Delta \phi / \Delta \varepsilon$, for the FFP of $\sim 0.57 \mathrm{rad} / \mu \varepsilon$. Data are recorded on a PC and the phase generated carrier demodulation method is used to extract the phase.

Results and discussion: Fig. 2 shows the AC strain resolution for two cores and the difference in strain demonstrating a differential strain noise of $0.6 \mathrm{n} \varepsilon / \mathrm{Hz}^{1 / 2}\left(340 \mu \mathrm{rad} / \mathrm{Hz}^{1 / 2}\right)$. This corresponds to a curvature resolution of $1.2 \times 10^{-5} \mathrm{~m}^{-1} / \mathrm{Hz}^{1 / 2}$. This is limited by beat noise due to residual back-reflection from the fan-out and can be reduced by eliminating the back-reflection. The tone at $10 \mathrm{~Hz}$ is a calibration tone applied through a piezoelectric fibre stretcher placed in one arm of the MI. The other tones are environmental acoustic and vibration noise and are mostly cancelled in the differential phase.

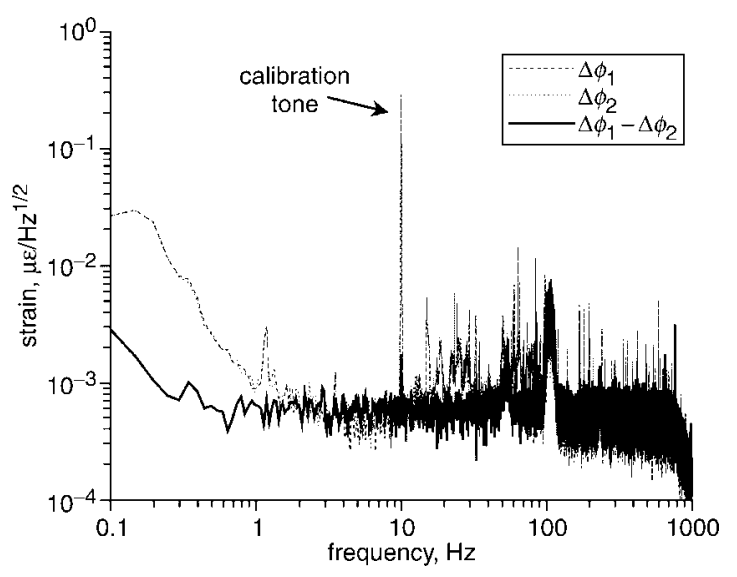

Fig. 2 Strain resolution of two cores and differential strain 
To characterise the response of the curvature sensor, the MCF is placed inside a metal tube of diameter $1.5 \mathrm{~mm}$. The tube is fixed at one end, forming a cantilever, and the other end is attached to an actuator that applies controlled bending in two orthogonal axes (see Fig. $3 a$ inset). Fig. $3 a$ shows the phase for two of the MCF-FFPs and the difference in phase, as the cantilever beam is moved in steps of $0.2 \mathrm{~mm}$ up to $2 \mathrm{~mm}$ first in the $y$-axis and then in the $x$-axis. The phase can be seen to wander erratically due to axial strain on the FFP and temperature changes. However, the difference in phase reveals a well-defined curvature related signal. The alignment of the MCF is found to be $1.9^{\circ}$ from the bending axes of the experimental setup. Fig. $3 b$ shows the difference in phase between two orthogonal sets of adjacent cores against displacement when curvature is applied parallel to the plane of each set of cores. Data points for both increasing and decreasing curvature are shown, illustrating excellent linearity and low hysteresis.
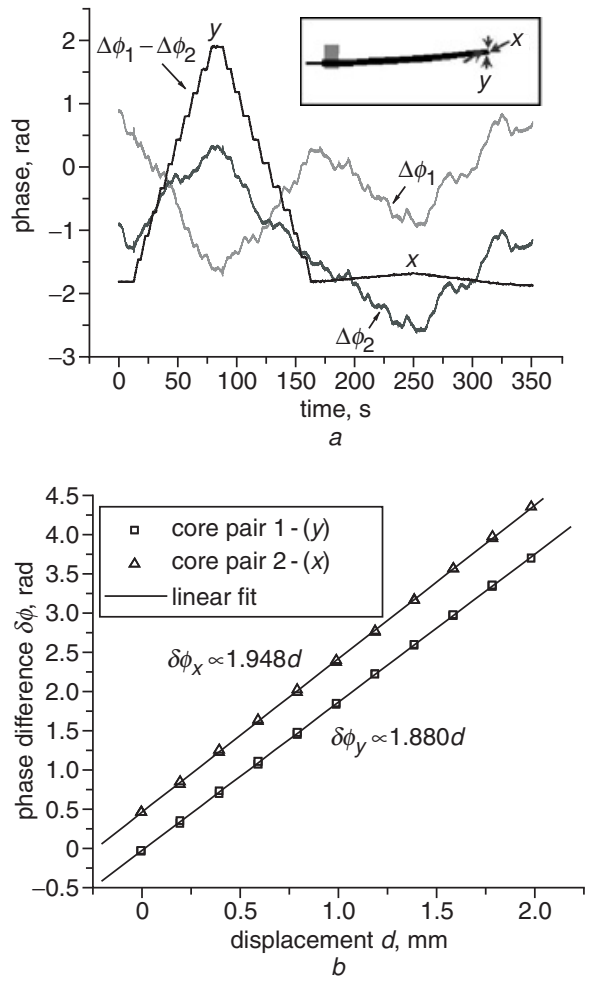

Fig. 3 Output response, and phase difference against displacement $a$ Output response for pair of cores to controlled bending in plane parallel to cores

$b$ Phase difference against displacement for two pairs of cores subjected to curvature in $x$ - and $y$-axes
This MCF-FFP curvature sensor demonstrates the highest curvature resolution reported to date from a fibre-optic sensor and would find application in ultra-high precision structural shape measurement.

Acknowledgments: The authors thank France Telecom for the provision of the multicore optical fibre, and W. N. MacPherson acknowledges funding from the UK EPSRC via the Advanced Fellowship scheme.

(C) The Institution of Engineering and Technology 2006

8 March 2006

Electronics Letters online no: 20060731

doi: 10.1049/el:20060731

G.A. Cranch and G.M.H. Flockhart (SFA Inc., Crofton, MD 21114, USA)

E-mail: geoff.cranch@nrl.navy.mil

W.N. MacPherson and J.S. Barton (Heriot-Watt University, School of Engineering \& Physical Sciences, Edinburgh, United Kingdom)

C.K. Kirkendall (Naval Research Laboratory, 4555 Overlook Avenue SW, Washington, DC 20375, USA)

G.A. Cranch: Also with Naval Research Laboratory, 4555 Overlook Avenue SW, Washington DC 20375, USA

\section{References}

1 Udd, E.: 'Fibre optic smart structures' (John Wiley \& Son, New York, 1995), Chap. 10

2 Flockhart, G.M.H., MacPherson, W.N., Barton, J.S., Jones, J.D.C., Zhang, L., and Bennion, I.: 'Two-axis bend measurement with Bragg gratings in multicore optical fiber', Opt. Lett., 2003, 28, (6), pp. 387-389

3 Kersey, A.D., Berkoff, T.A., and Morey, W.W.: 'Fiber optic Bragg grating strain sensor with drift-compensated high-resolution interferometric wavelength-shift detection', Opt. Lett., 1993, 18, (1), pp. 72-74

4 Flockhart, G.M.H., Cranch, G.A., and Kirkendall, C.K.: 'Differential phase tracking applied to Bragg gratings in multicore fibre for high accuracy curvature measurement', Electron. Lett., 2006, 42, (7), pp. $17-18$

5 Cranch, G.A., Flockhart, G.M.H., and Kirkendall, C.K.: 'Efficient fiber Bragg grating and fiber Fabry-Perot sensor multiplexing scheme using a broadband, pulsed mode-locked laser', J. Lightwave Technol, 2005, 23, (11), pp. 3798-3807

6 Bethuys, S., Lablonde, L., Rivoallan, L., and Auvray, P.: ' $1 \times 4$ fused multicore singlemode fibre coupler: theory, fabrication and analysis', Electron. Lett., 1998, 34, (15), pp. 1516-1517 